\title{
PADRÕES DE DISTRIBUIÇÃO VOLUMÉTRICA DE PONTAS DE PULVERIZAÇÃO DE JATO PLANO 11002, COM E SEM INDUÇÃO DE AR, SOB DIFERENTES ESPAÇAMENTOS E ALTURAS
}

\section{FERNANDO C. BAUER ${ }^{1}$, CARLOS G. RAETANO ${ }^{2}$, FRANCISCO DE A. R. PEREIRA ${ }^{2}$}

RESUMO: O presente trabalho teve como objetivo avaliar a distribuição volumétrica de pontas de pulverização hidráulica de jato de uso ampliado 11002, com e sem indução de ar, em laboratório, bem como o padrão de deposição da pulverização, por meio da estimativa do coeficiente de variação (C.V.) obtido pela simulação da sobreposição de jatos adjacentes. As pontas foram posicionadas, isoladamente, no centro da mesa de teste, a 30; 40 e $50 \mathrm{~cm}$ de altura da mesa e a 300 e $500 \mathrm{kPa}$ de pressão. Foram avaliadas 20 unidades de cada tipo de ponta, e a deposição média foi utilizada para a simulação da deposição ao longo da barra pulverizadora, com as pontas espaçadas em 30; 40; 50 e 60 cm entre si. A uniformidade da distribuição foi estimada pelo cálculo do C.V. resultante da simulação da sobreposição das pontas em barra de $8 \mathrm{~m}$, sendo utilizados somente os $4 \mathrm{~m}$ centrais no cálculo do C.V. Os resultados mostraram haver diferenças relacionadas à deposição entre os dois tipos de ponta. A ponta com indução de ar resultou em área de deposição inferior à ponta sem indução de ar. Esse comportamento foi observado em todas as alturas da barra e nas duas pressões, podendo-se inferir que esse comportamento possa ser característico das pontas com indução de ar.

PALAVRAS-CHAVE: tecnologia de aplicação, pulverizador de barra, indução de ar.

\section{DISTRIBUTION PATTERN OF 11002 FLAT FAN NOZZLES, WITH AND WITHOUT AIR INDUCTION, UNDER DIFFERENT OPERATIONAL CONDITIONS}

\begin{abstract}
The spray distribution and uniformity characteristics of 11002 flat fan nozzles type with and without air induction were evaluated in the present research. Twenty units of flat fan nozzles with and without air induction were tested in patternator table. Each one of the units was tested separately, at the 30; 40 and $50 \mathrm{~cm}$ above the patternator table and submitted to pressures of 300 and $500 \mathrm{kPa}$. The means of the colleted spray volume distribution of each nozzle type was evaluated and entered in a PC program. Based on the deposition patterns, distribution uniformity for nozzles spaced at 30;40;50 and $60 \mathrm{~cm}$ was simulated in PC Program. The distribution uniformity was estimated by the variation coefficient of the deposition simulated for boom with 8 meters. The PC Program simulated the distribution pattern of the boom and calculated the coefficient of variation, corresponding to each configuration. The results pointed out different deposition for each nozzle type. The nozzle with air induction resulted in lower deposition area. The behavior was observed in all the heights and two pressures. Can be inferred that behavior would be characteristic of the tip with air induction.
\end{abstract}

KEYWORDS: application technology, air induction nozzles, boom sprayer.

\section{INTRODUÇÃO}

A utilização dos produtos fitossanitários implica, obrigatoriamente, sua colocação no ambiente de forma e em quantidade corretas. Para isso, todos os produtos devem passar por processo de aplicação que assume, então, vital importância. Por isso, o domínio da tecnologia de aplicação mostra-se fundamental para o aumento da eficiência do produto e a diminuição da

\footnotetext{
${ }^{1}$ Eng $\mathrm{o}$ Agrônomo, Prof. Dr., Universidade para o Desenvolvimento do Estado e da Região do Pantanal - UNIDERP, Campo Grande - MS, fcbauer@mail.uniderp.br

${ }^{2}$ Prof. Dr., Departamento de Produção Vegetal, UNESP, Botucatu - SP.

Recebido pelo Conselho Editorial em: 25-4-2006
}

Aprovado pelo Conselho Editorial em: 10-8-2006 
contaminação do aplicador e do ambiente, bem como para a redução dos custos nas aplicações (BAUER \& PEREIRA, 2005). Muitos são os aspectos que interferem negativamente na qualidade da pulverização, destacando-se, entre eles, aspectos relacionados às pontas de pulverização. Essas peças são consideradas as mais importantes do pulverizador, por serem as responsáveis por diversos aspectos relacionados à qualidade da aplicação, como tamanho das gotas, distribuição do líquido pulverizado, uniformidade de distribuição e volume de calda (BAUER \& RAETANO, 2004a).

Com a necessidade de redução nas doses e volume de aplicação, para diminuição do custo de produção, a uniformidade na distribuição da pulverização deve ser alcançada, caso contrário a dose que chega ao alvo poderá ser insuficiente para causar o efeito biológico desejável. Para atender a essa necessidade, houve grande evolução dos equipamentos de aplicação, inclusive das pontas, hoje com diversas características de pulverização, distribuição e tamanho de gotas, incluindo as mais recentes com indução de ar. Essa nova tecnologia, indução de ar, vem sendo muito utilizada em pulverizações no Centro-Oeste, pois resulta em menor interferência das condições climáticas, sem alteração significativa na taxa de aplicação, mas com formação de gotas de diâmetros maiores.

A uniformidade na distribuição da calda aplicada, em pulverizações de produtos fitossanitários, é dada pelas condições de montagem e de operação do equipamento, como espaçamento entre bicos, altura da barra, ângulo de abertura dos jatos e pressão de trabalho (PERECIN et al., 1994).

MATTHEWS (2000) afirma que cada ponta possui característica própria de distribuição volumétrica e que essa curva tem grande importância na determinação da altura do bico em relação ao alvo e no espaçamento entre bicos na barra, devendo haver sobreposição do jato de um bico com os adjacentes para conseguir distribuição uniforme do líquido pulverizado.

Uma das formas de quantificar a uniformidade de distribuição numa pulverização é por meio da análise da deposição volumétrica do produto na área, que é expressa pelo coeficiente de variação (C.V.) obtido dessa análise; quanto menor esse valor, menos desuniforme é a distribuição (CHRISTOFOLETTI, 1997). Dessa forma, aplicação com C.V. igual a zero indicaria distribuição e uniformidade perfeitas, ou seja, a mesma quantidade de líquido depositado ao longo de toda a barra pulverizadora, sem variações. Na Itália, o registro, aprovação, comercialização e certificação de máquinas novas têm como base C.V. abaixo de 7\% (CONAMA, 1997). No Brasil, porém, não há normas para comercialização, registro ou certificação de pulverizadores.

Normalmente, o C.V. é calculado a partir do padrão de distribuição de bicos individuais, simulando-se, então, a sobreposição desses padrões. Assim, os padrões de deposição individual dos bicos são determinados em bancadas de prova, nas quais canaletas realizam a coleta do líquido a distâncias determinadas e o depositam em recipientes individuais (CHAPPLE et al., 1993).

Assim, o presente trabalho teve como objetivo determinar, em laboratório, o padrão de distribuição, em duas pressões e três alturas de barra, de pontas de jato plano 11002 com e sem indução de ar.

\section{MATERIAL E MÉTODOS}

O trabalho foi desenvolvido na Faculdade de Ciências Agronômicas da Universidade Estadual Paulista, Câmpus de Botucatu, no Laboratório de Tecnologia de Aplicação de Defensivos Agrícolas do Departamento de Produção Vegetal - Defesa Fitossanitária.

Uma mesa de testes para bicos hidráulicos foi construída de acordo com as Normas ISO 5.682-1:1996. A mesa consta de 60 canaletas de alumínio de $5 \mathrm{~cm}$ de largura e $200 \mathrm{~cm}$ de comprimento cada, totalizando $300 \mathrm{~cm}$ x $200 \mathrm{~cm}$ de área útil de coleta. Ao final de cada canaleta conectou-se um tubo de drenagem que conduz o líquido a uma proveta graduada e numerada, de acordo com a canaleta a que está conectada. 
A barra pulverizadora, onde os bicos foram fixados, é independente da mesa, em forma de "U" invertido, com o travessão ficando a $230 \mathrm{~cm}$ de altura, porém sendo possível sua regulagem a cada $5 \mathrm{~cm}$, podendo-se trabalhar com a barra desde 10 até $150 \mathrm{~cm}$ de altura em relação à superfície da mesa.

O sistema funciona com energia proveniente de motor elétrico trifásico com potência de $2,9 \mathrm{~kW}(4 \mathrm{cv})$, que, por meio de correia, movimenta a bomba de três pistões marca Jacto, com vazão de $42 \mathrm{~L} \mathrm{~min}^{-1}$ e pressão máxima de $3.431 \mathrm{kPa}$. No momento dos testes, no interior do laboratório, a umidade relativa do ar variou entre 67 e $85 \%$, e a temperatura, entre 24 e $26{ }^{\circ} \mathrm{C}$.

Foram adquiridas no mercado, 20 unidades das pontas de pulverização de jato plano de uso ampliado (AXI 11002) e 20 unidades da ponta (AVI 11002) de jato plano comum com indução de ar. Segundo o fabricante, às pressões utilizadas, as pontas (AXI 11002) produzem gotas finas e as pontas (AVI 11002) grossas a muito grossas, segundo a Norma ASAE S572. Cada uma das 20 unidades das pontas foi instalada, isoladamente, nas alturas de 30; 40 e $50 \mathrm{~cm}$ em relação à superfície da mesa, na posição vertical e, em cada altura, submetidas às pressões de 300 e $500 \mathrm{kPa}$. Cada ponta, considerada como uma repetição, foi instalada centrada na mesa e posta em funcionamento por dois minutos; a partir daí, iniciava-se a coleta do líquido pulverizado por um minuto, quando, então, encerrava-se a coleta, momento em que se fazia a leitura dos volumes de cada proveta. Em todas as aplicações, a calda utilizada foi composta somente de água.

Baseado nos volumes médios coletados nas 20 repetições, em cada tratamento, foram determinados os perfis médios de distribuição volumétrica, possibilitando o cálculo do padrão médio de distribuição ao longo da barra pulverizadora, determinado em programa Microsoft Excell, no qual se fez a simulação da sobreposição dos bicos numa barra hipotética de $8 \mathrm{~m}$, sendo utilizados somente os $4 \mathrm{~m}$ centrais para os cálculos do coeficiente de variação (C.V.) abaixo da barra.

Avaliou-se a distribuição da pulverização pelo coeficiente de variação obtido pela eq.(1):

C.V. = (desvio-padrão/média) 100

Essa equação foi aplicada em espaçamentos de 30; 40; 50 e $60 \mathrm{~cm}$ com a barra pulverizadora a 30; 40 e $50 \mathrm{~cm}$ de altura. Esse procedimento e outros similares já foram validados por PERECIN et al. (1998), BAUER \& RAETANO (2004a) e FREITAS et al. (2005).

A vazão das pontas utilizadas foi estimada por meio da coleta do líquido pulverizado, por todas as pontas, individualmente, com o uso de proveta inserida logo abaixo da barra, promovendo coleta de $100 \%$ da pulverização. Os valores foram comparados à vazão informada pelo fabricante, e as pontas onde a vazão apresentou variação de $5 \%$, tanto para mais quanto para menos do recomendado pelo fabricante, foram excluídas dos testes.

\section{RESULTADOS E DISCUSSÃO}

Os resultados obtidos com os testes de vazão indicaram valores próximos à vazão recomendada, sendo os valores médios apresentados na Tabela 1. Observa-se que os valores se encontram dentro dos padrões de utilização em relação àqueles indicados pelo fabricante, embora todos estejam aplicando volumes a mais do indicado. Isso indica que a vida útil das pontas será diminuída, pois a recomendação é que sejam substituídas quando a vazão for $10 \%$ acima do recomendado pelo fabricante. Como as pontas novas já iniciam o trabalho aplicando volume superior ao recomendado, sua vida útil será reduzida.

Os dados de deposição, obtidos na mesa de teste, foram utilizados sob a forma de percentagem do volume total aplicado.

BAUER \& RAETANO (2004b) afirmam ser o coeficiente de variação (C.V.) sob a barra mais importante que o volume depositado, pois poderá haver volumes diferentes de depósitos com coeficientes de variação idênticos; por isso, aqui, optou-se por avaliar somente o padrão de distribuição da ponta e o C.V. da deposição e seus efeitos na sobreposição das pontas na barra. 
TABELA 1. Relações entre a vazão nominal e vazão observada em pontas de pulverização jato planto convencional e com indução de ar, instaladas em mesa para teste de deposição.

\begin{tabular}{|c|c|c|c|c|c|}
\hline $\begin{array}{l}\text { Ponta Jato } \\
\text { Plano }\end{array}$ & $\begin{array}{c}\text { Pressão } \\
(\mathrm{kPa})\end{array}$ & $\begin{array}{l}\text { Vazão Observada (1) } \\
(\mathrm{L} \text { min })^{-1}\end{array}$ & $\begin{array}{l}\text { Vazão-Padrão (2) } \\
\left(\mathrm{L} \mathrm{min}^{-1}\right)\end{array}$ & $\begin{array}{l}\text { Diferença 1-2 } \\
\left(\mathrm{L} \mathrm{min}^{-1}\right)\end{array}$ & $\begin{array}{c}\text { Diferença } 2-1 \\
(\%)\end{array}$ \\
\hline \multirow{2}{*}{$\begin{array}{c}11002 \text { com } \\
\text { indução de ar }\end{array}$} & 300 & 0,82 & 0,79 & 0,03 & $+3,79$ \\
\hline & 500 & 1,03 & 1,02 & 0,01 & $+0,98$ \\
\hline \multirow{2}{*}{$\begin{array}{c}11002 \\
\text { convencional }\end{array}$} & 300 & 0,82 & 0,79 & 0,03 & $+3,79$ \\
\hline & 500 & 1,06 & 1,02 & 0,04 & $+3,92$ \\
\hline
\end{tabular}

Os perfis de distribuição das pontas com e sem indução de ar, para as diferentes alturas e pressões, encontram-se nas Figuras 1 e 2. Puderam-se observar efeitos similares em ambas as pontas quando se altera a altura da barra. Há, porém, diferenças relacionadas à largura da deposição entre as diferentes pontas, pois a ponta com indução de ar à pressão de $300 \mathrm{kPa}$ obteve cobertura de $90 \mathrm{~cm}$, enquanto, para a ponta sem indução, à mesma pressão, a deposição foi de $130 \mathrm{~cm}$, a $50 \mathrm{~cm}$ de altura.



FIGURA 1. Perfis de distribuição volumétrica de pontas de jato plano comum, com e sem indução de ar, submetidas à pressão de $300 \mathrm{kPa}$ e $30 ; 40$ e $50 \mathrm{~cm}$ de altura, com largura da faixa de deposição e distribuição do volume (\%) obtidas em mesa de teste para pontas de pulverização hidráulica.

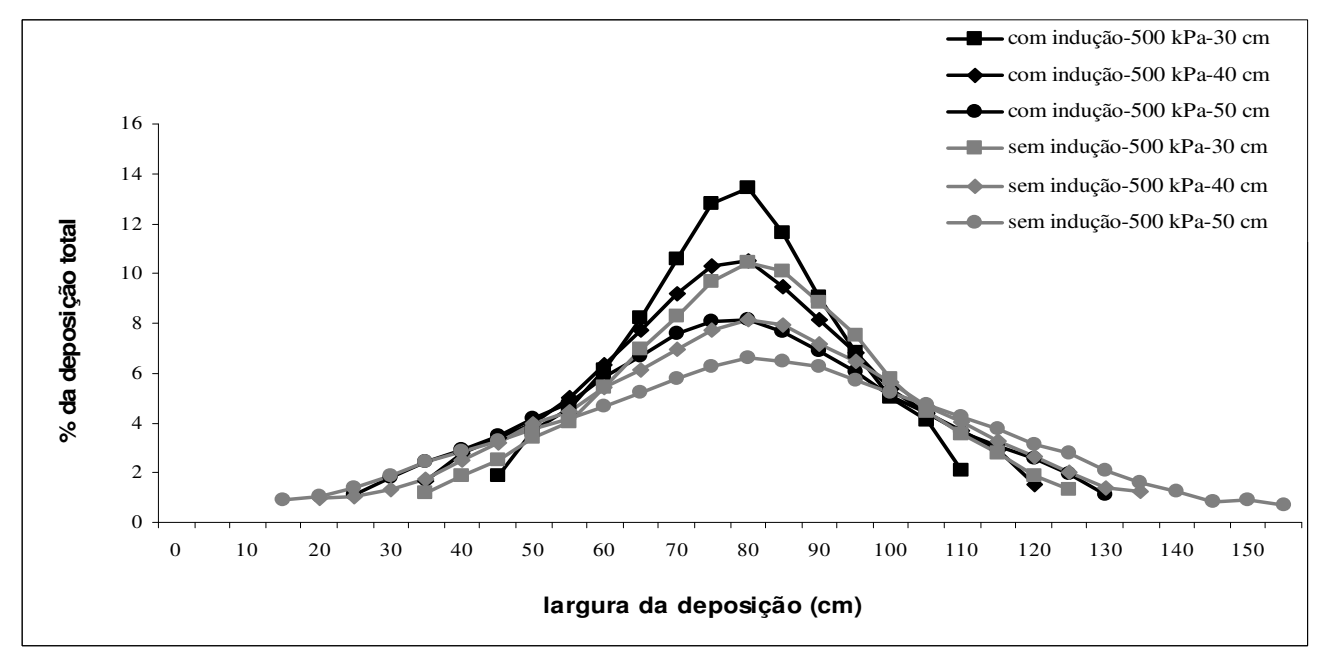

FIGURA 2. Perfis de distribuição volumétrica de pontas de jato plano comum, com e sem indução de ar, submetidas à pressão de $500 \mathrm{kPa}$ e $30 ; 40$ e $50 \mathrm{~cm}$ de altura, com largura da faixa de deposição e distribuição do volume (\%) obtidas em mesa de teste para pontas de pulverização hidráulica. 
Esse comportamento foi observado em todas as alturas da barra para as duas pressões (Figuras 1 e 2), podendo-se inferir que, devido à produção de gotas maiores, as pontas com indução de ar tenham comportamento diferente das pontas sem indução de ar. Isso tem efeito na sobreposição das pontas, sendo demonstrado na Tabela 2, em que se pode observar a percentagem de sobreposição entre as diferentes pontas para mesma pressão e altura de barra.

TABELA 2. Coeficiente de variação (C.V.) e sobreposição dos bicos (\%) obtidos em simulação da pulverização com pontas AVI e AXI 11002 a quatro espaçamentos entre bicos, duas pressões e três alturas de barra.

\begin{tabular}{|c|c|c|c|c|c|c|c|c|}
\hline \multirow{3}{*}{ Ponta } & \multirow{3}{*}{$\begin{array}{c}\text { Pressão } \\
(\mathrm{kPa})\end{array}$} & \multirow{3}{*}{$\begin{array}{c}\text { Espaçamento } \\
\text { entre Bicos } \\
(\mathrm{cm})\end{array}$} & \multicolumn{6}{|c|}{ Altura da Barra $(\mathrm{cm})$} \\
\hline & & & \multicolumn{2}{|r|}{30} & \multicolumn{2}{|c|}{40} & \multicolumn{2}{|r|}{50} \\
\hline & & & $\begin{array}{l}\text { C.V. } \\
(\%)\end{array}$ & $\begin{array}{c}\text { Sobreposição } \\
(\%)\end{array}$ & $\begin{array}{l}\text { C.V. } \\
(\%)\end{array}$ & $\begin{array}{c}\text { Sobreposição } \\
(\%)\end{array}$ & $\begin{array}{l}\text { C.V. } \\
(\%)\end{array}$ & $\begin{array}{c}\text { Sobreposição } \\
(\%)\end{array}$ \\
\hline \multirow{8}{*}{ AVI 11002} & \multirow{4}{*}{300} & 30 & 10,1 & 54 & 12,3 & 63 & 10,8 & 67 \\
\hline & & 40 & 4,5 & 38 & 4,7 & 50 & 9,6 & 56 \\
\hline & & 50 & 6,9 & 23 & 4,5 & 38 & 6,6 & 44 \\
\hline & & 60 & 23,7 & 7,7 & 17,0 & 25 & 7,3 & 33 \\
\hline & \multirow{4}{*}{500} & 30 & 9,6 & 57 & 8,9 & 67 & 10,2 & 73 \\
\hline & & 40 & 6,6 & 43 & 8,6 & 56 & 9,1 & 64 \\
\hline & & 50 & 19,2 & 29 & 5,5 & 44 & 6,2 & 55 \\
\hline & & 60 & 36,4 & 14 & 44,5 & 33 & 4,3 & 45 \\
\hline \multirow{8}{*}{ AXI 11002- } & \multirow{4}{*}{300} & 30 & 8,8 & 65 & 9,3 & 71 & 11,0 & 77 \\
\hline & & 40 & 6,9 & 53 & 8,7 & 62 & 8,6 & 69 \\
\hline & & 50 & 6,7 & 41 & 4,7 & 52 & 7,0 & 62 \\
\hline & & 60 & 16,8 & 29 & 3,7 & 43 & 4,8 & 54 \\
\hline & \multirow{4}{*}{500} & 30 & 8,5 & 68 & 10,2 & 75 & 11,5 & 79 \\
\hline & & 40 & 8,7 & 58 & 7,7 & 67 & 8,6 & 71 \\
\hline & & 50 & 8,5 & 47 & 6,4 & 58 & 7,0 & 64 \\
\hline & & 60 & 14,4 & 37 & 5,2 & 50 & 4,8 & 57 \\
\hline
\end{tabular}

O comportamento do C.V., em relação às condições testadas, pode ser visto na Tabela 2. Percebe-se que, embora com espaçamento e altura iguais, há diferenças na sobreposição, sem que isso resulte em diferenças significativas no C.V. Assim, a ponta com indução de ar, a $300 \mathrm{kPa}$, $30 \mathrm{~cm}$ de altura de barra e $40 \mathrm{~cm}$ de espaçamento obteve C.V. igual a 4,5\%, enquanto a ponta sem indução obteve C.V. igual a 6,9\%. Ambas se mantêm abaixo dos 7\%, embora haja pequena diferença. Valores abaixo de $7 \%$ são utilizados para registro, aprovação e certificação de equipamentos de pulverização agrícola na Itália (CONAMA, 1997).

Fator interessante, e que deve ser observado, relaciona-se à sobreposição entre as pontas na barra. A recomendação é que ocorra $30 \%$ de sobreposição entre as pontas para obter boa distribuição abaixo da barra pulverizadora. Entretanto, ao se observar a Tabela 2, percebe-se que ambas as pontas, em altura e espaçamento de $50 \mathrm{~cm}$, nas duas pressões trabalhadas, obtiveram valores bem próximos ou no limite recomendado de $7 \%$, mas com sobreposição sempre superior aos $30 \%$ recomendados. Isso ocorreu de forma geral em ambas as pontas, para as duas pressões e em todas as alturas e espaçamentos.

Valores de C.V. excessivamente altos foram obtidos com as pontas com indução de ar que, operadas a espaçamentos de $60 \mathrm{~cm}$ e $500 \mathrm{kPa}$, resultaram em C.V. igual a 36,4 e 44,5\% a 30 e $40 \mathrm{~cm}$ de altura, respectivamente. Embora com valores não tão altos, esse comportamento foi observado também à pressão de $300 \mathrm{kPa}$. Esses fatos podem indicar maior sensibilidade das pontas com indução de ar às variações na sobreposição, em relação às pontas sem indução.

Para altura de barra igual a $50 \mathrm{~cm}$, as únicas condições, em ambas as pontas, com C.V. até $7 \%$, foram quando o espaçamento entre bicos foi de $50 \mathrm{~cm}$, nas duas pressões. No entanto, ao contrário da conclusão de BAUER \& RAETANO (2004a), espaçamentos menores não obtiveram 
C.V. aceitável, ao passo que, ao utilizar $60 \mathrm{~cm}$ entre bicos, os CV ficaram abaixo de $7 \%$ para a altura de $50 \mathrm{~cm}$. Esse fato corrobora a afirmação de FREITAS et al. (2005) que, embora trabalhando com pontas diferentes, obtiveram dados indicativos de que maiores espaçamentos podem ser utilizados com bons resultados na distribuição da pulverização.

\section{CONCLUSÕES}

As aplicações com pontas com indução de ar resultaram em área de deposição, abaixo da barra, inferior às pontas sem indução de ar, indicando a necessidade de maior cuidado com a sobreposição da pulverização entre essas pontas.

Esse comportamento foi observado em todas as alturas de barra e pressões testadas, podendose inferir ser característico das pontas com indução de ar.

\section{REFERÊNCIAS}

BAUER, F.C.; PEREIRA, F.A.R. Fitossanidade e produção agrícola. In: BAUER, F.C.; VARGAS JUNIOR, F.M. (Coord.) Produção e gestão agroindustrial. Campo Grande: Editora Uniderp, 2005. p.23-48 p.

BAUER, F.C.; RAETANO, C.G. Distribuição volumétrica de calda produzida pelas pontas de pulverização XR, TP e TJ sob diferentes condições operacionais. Planta Daninha, Viçosa, v.22, n.2, p.275-84, 2004a.

BAUER, F.C; RAETANO, C.G. Perfis de distribuição volumétrica de pontas XR 11003 e TXVK-4 em diferentes condições de pulverização. Engenharia Agrícola, Jaboticabal, v.24, n.2, p.364-73, 2004b.

CHAPPLE, A.C.; HALL, F.R.; BISHOP, B.L. Assessment of single-nozzle patternation and extrapolation to moving booms. Crop Protection, Oxford, v.12, p.207-13, 1993.

CHRISTOFOLETTI, J.C. Bicos de pulverização - seleção e uso. Diadema: Spraying Systems do Brasil, 1997. 8 p.

CONAMA. Consorzio Nazionale per la Meccanizzazione Agricola. Roma, 1997. 11 p. (Circolare Ministeriale 10189)

FREITAS, F.C.L.; TEIXEIRA, M.M.; FERREIRA, L.R.; FERREIRA, F.A.; MACHADO, A.F.L.; VIANA, R.G. Distribuição volumétrica de pontas de pulverização turbo teejet 11002 em diferentes condições operacionais. Planta Daninha, Viçosa, v.23, n.1, p.161-7, 2005.

INTERNATIONAL ORGANIZATION FOR STANDARDIZATION. ISO 5682-1: Equipment for crop protection - Spraying equipment - Part 1: Test methods for sprayer nozzles. Genebra, 1997. 22 p. (International Standard ISO 5682-1:1996(E)).

MATTHEWS, G.A. Pesticide application methods. $3^{\text {th }}$ ed. London: Blackwell, 2000. 432 p.

PERECIN. D.; PERESSIN, V.A.; MATUO, T.; BARBOSA, J.C.; PIO, L.C, BRAZ, B.A. Padrões de distribuição obtidos com bicos Twinjet em função da altura e do espaçamento entre bicos. Engenharia Agrícola, Jaboticabal, v.14, p.19-30, 1994.

PERECIN, D.; PERESSIN, V.A.; MATUO, T.; BRAZ, B.A.; PIO, L.C. Padrões de distribuição obtidos com bicos TF-4, TJ60-11006 e TQ15006 em mesa de prova. Pesquisa Agropecuária Brasileira, Brasília, v.33, n.2, p.175-182, 1998. 